\title{
Evolution of the chromosomal location of rDNA genes in two Drosophila species subgroups: ananassae and melanogaster
}

\author{
V Roy ${ }^{1,4}$, L Monti-Dedieu ${ }^{1,2,4}$, N Chaminade ${ }^{1}$, S Siljak-Yakovlev ${ }^{3}$, S Aulard ${ }^{1}$, F Lemeunier ${ }^{1}$ \\ and C Montchamp-Moreau ${ }^{1}$ \\ ${ }^{1}$ Laboratoire Populations, Génétique et Evolution, Centre National de la Recherche Scientifique, Gif-sur-Yvette Cedex, France; ${ }^{2}$ Institut \\ Universitaire de Formation des Maîtres, Paris, France; ${ }^{3}$ Laboratoire d'Evolution et Systématique, Université Paris-Sud XI, Centre \\ National de la Recherche Scientifique, Orsay Cedex, France
}

\begin{abstract}
The evolution of the chromosomal location of ribosomal RNA gene clusters and the organization of heterochromatin in the Drosophila melanogaster group were investigated using fluorescence in situ hybridization and DAPI staining to mitotic chromosomes. The investigation of 18 species (11 of which were being examined for the first time) belonging to the melanogaster and ananassae subgroups suggests that the ancestral configuration consists of one nucleolus organizer (NOR) on each sex chromosome. This pattern, which is conserved throughout the melanogaster subgroup, except in $D$. simulans and $D$. sechellia, was observed only in the ercepeae complex within the ananassae subgroup. Both sexlinked NORs must have been lost in the lineage leading to
\end{abstract}

D. varians and in the ananassae and bipectinata complexes, whereas new sites, characterized by intra-species variation in hybridization signal size, appeared on the fourth chromosome related to heterochromatic rearrangements. Nucleolar material is thought to be required for sex chromosome pairing and disjunction in a variety of organisms including Drosophila. Thus, either remnant sequences, possibly intergenic spacer repeats, are still present in the sex chromosomes which have lost their NORs (as observed in $D$. simulans and $D$. sechellia), or an alternative mechanism has evolved.

Heredity (2005) 94, 388-395. doi:10.1038/sj.hdy.6800612

Published online 23 February 2005

Keywords: Drosophila; rDNA; heterochromatin; evolution; fluorescence in situ hybridization

\section{Introduction}

In all eukaryotes, the genes encoding ribosomal RNA (rDNA) are present in multiple copies, arranged as clusters embedded in heterochromatin and cytologically visible as nucleolus organizers (NORs) when they are transcriptionally active. Typically, each repeat unit within an rDNA locus contains a gene region encoding the 18S, 5.8S and 28S rRNAs, and an intergenic spacer consisting of different subrepeats. Investigation of a wide range of organisms, including fungi, animals and plants, has revealed that the organization, size and degree of repetition of the basic unit are highly variable (reviewed in John and Miklos, 1988). Changes in the chromosomal position and number of NORs have sometimes been reported between closely related species, and even within a given species (Andronico et al, 1985; Dubcovsky and Dvorak, 1995; Galian et al, 1995; Hirai et al, 1996; Shishido et al, 2000), showing that these genes can undergo rapid rearrangements due to their heterochromatic location. It follows that studying rDNA genes

Correspondence: L Monti-Dedieu, Laboratoire Populations, Génétique et Evolution, Centre National de la Recherche Scientifique, Avenue de la Terrasse, 91198 Gif-sur-Yvette Cedex, France. E-mail: monti@paris.iufm.fr ${ }^{4}$ These authors contributed equally to the work.

Received 22 May 2003; accepted 30 July 2004; published online 23 February 2005 should help us to understand how genes evolve in the heterochromatin, and also to resolve the phylogeny of closely related species.

Data on the number and chromosomal locations of NORs are very fragmentary in the genus Drosophila, which includes about 3500 known species and which is thought to have originated $61-65$ million years ago (Powell, 1997). They are only available for 20 species, scattered across three of the 15 subgenera. The two members of the Idiomyia subgenus studied, D. heteroneura and D. sylvestris, exhibit only autosomal NORs (Stuart et al, 1981), whereas, in most of the species examined in the Sophophora and Drosophila subgenera, the NORs appear to be restricted to the sex chromosomes. In the subgenus Drosophila and in the repleta group, both $D$. repleta and D. hydei, as well as two of their closest relatives, possess one NOR on the $X$ chromosome and two on the Y chromosome (Hennig et al, 1975, 1982). One of these species also possesses rDNA sequence clusters on several autosomes, but these appear to be transcriptionally inactive. Again in the repleta group, molecular and genetic data indicate that an NOR is present on each sex chromosome of $D$. mercatorum and D. buzzatii (Templeton et al, 1985; Knibb et al, 1989). The arrangement is different in the sibling species $D$. arizonensis and D. mulleri, which possess only an X-linked NOR together with a cluster of not very active rDNA genes on the dot chromosome (Bicudo, 1981). In D. tumiditarsus, a single 
NOR is found on the dot chromosome (Sinibaldi and Cummings, 1981). In the Sophophora subgenus, eight species belonging to the melanogaster subgroup have been examined. All of these have an NOR on each sex chromosome, with the exception of $D$. simulans and $D$. sechellia, which were found to possess only an Xlinked NOR (Lohe and Roberts, 2000). However, the $Y$ chromosome of $D$. simulans has been shown to carry a large block of 240-bp nontranscribed spacer repeats (Lohe and Roberts, 1990). The only other species studied in the Sophophora subgenus, D. ananassae (ananassae subgroup), carries NORs on its $\mathrm{Y}$ and fourth chromosomes (Kaufmann, 1937; Kikkawa, 1938).

To get a more precise picture of how the rDNA clusters have evolved at the species group and subgroup levels in Drosophila, we used fluorescence in situ hybridization (FISH) on mitotic chromosomes, to add to the data on the melanogaster and ananassae subgroups. In the melanogaster subgroup, the phylogenetic relationships are clear, except for the simulans-sechellia-mauritiana triad. In the ananassae subgroup, morphological, molecular, karyotypic and behavioural data strongly support a division into three complexes ananassae, bipectinata and ercepeae (Bock and Wheeler, 1972; Lemeunier et al, 1986, 1997; Schawaroch, 2002; Da Lage, personal communication). However, the phylogenetic relationships of the complexes have not been resolved, nor is the positioning of D. varians, a species that does not seem to fit into any of these complexes. To shed some light on these issues, we examined $D$. varians and 11 other species chosen from the three complexes.

\section{Materials and methods}

Drosophila strains and chromosome spreads

The species studied in the melanogaster and ananassae subgroups are listed in Table 1. Mitotic chromosomes were obtained from the brain of last-instar larvae using conventional procedures: colchicine and hypotonic treatments, fixation in methanol:acetic acid (3:1) and spreading with $60 \%$ acetic acid. Slides were stored in the freezer until use. Between three and six individuals were analysed for each species in the ananassae and melanogaster subgroups, except for D. simulans, for which 11 individuals were studied.

\section{Fluorescence in situ hybridization}

The rDNA probe used in the study was Py12 isolated from D. melanogaster and cloned in the PMB9 plasmid ( $\mathrm{R}$ Terracol, Institut J Monod, Paris). It contained a complete DNA array unit, that is, the 18S, 5.8S and $28 \mathrm{~S}$ genes, and the intergenic nontranscribed spacers. A probe containing the 240-bp nontranscribed spacer repeats from $D$. simulans cloned in the A3235PS plasmid (Lohe and Roberts, 1990) was also used. Both probes were labelled with digoxygenin-11-dUTP (Roche Diagnostics) by nick translation.

The slides were pretreated in $2 \times$ SSC at $37^{\circ} \mathrm{C}$ and dehydrated. The probe solution was prepared in $2 \times$ SSC, $35 \%$ formamide and 10\% dextran sulphate. It was then deposited on the slides $(10 \mu \mathrm{l}$ per slide) and covered with a glass coverslip. Chromosome and probe DNA were simultaneously denatured for $5 \mathrm{~min}$ at $72^{\circ} \mathrm{C}$ on a hot plate. Hybridization was performed at $37^{\circ} \mathrm{C}$ overnight in a humidified chamber. After removing the glass coverslip, the slides were washed for $15 \mathrm{~min}$ at room temperature in a $50 \%$ formamide $/ 2 \times$ SSC solution, and then washed in $2 \times$ SSC and $1 \times$ PBD (Q-biogene). Fluorescence detection consisted of incubating the slides for $5 \mathrm{~min}$ with rhodamine anti-digoxygenin $(60 \mu \mathrm{l}$ per slide) (Q-biogene) followed by washes in $1 \times \mathrm{PBD}$, counterstaining and mounting in DAPI/Antifade (Q-biogene).

Slides were observed under a Zeiss Axioplan 2 epifluorescence microscope. Chromosome images were

Table 1 Species and strains used in this study

\begin{tabular}{lll}
\hline Species & Origin & Reference of the stock \\
\hline melanogaster subgroup & & \\
D. melanogaster & Chaland Is. (Mauritius) & Canton-S, PGE laboratory \\
D. mauritiana & Seychelles & PGE laboratory, No. 163-1 \\
D. sechellia & Tunisia & PGE laboratory, No. 228 \\
D. simulans & Zimbabwe & PGE laboratory, No. SR \\
D. teissieri & São Tomé Is. & PGE laboratory, No. 128-2 \\
D. santomea & Cameroon & PGE laboratory \\
D. yakuba & & PGE laboratory, No. 115 \\
& & \\
ananassae subgroup & & \\
(1) ananassae complex & Ivory Coast & \\
D. ananassae & Thailand & PGE laboratory, No. 242-1 \\
D. atripex & Samoa & PGE laboratory \\
D. pallidosa & Fiji & Tucson Stock centre, No. 14024-0433-1 \\
D. phaeopleura & Tucson Stock centre, No. 14024-0434-0 \\
(2) bipectinata complex & Pew Caledonia & \\
D. bipectinata & PGE laboratory \\
D. malerkotliana & Ivory Coast & PGE laboratory, No. 243-1 \\
D. parabipectinata & Mauritius & PGE laboratory \\
D. pseudoananassae & Thailand & Tucson Stock centre, No. 14024-0421-0 \\
(3) ercepeae complex & & \\
D. ercepeae & La Réunion & PGE laboratory, No. 164-14 \\
D. merina & Madagascar & PGE laboratory, No. 290-1 \\
D. vallismaia & Seychelles & PGE laboratory, No. 206-11 \\
(4) D. varians & Philippines & PGE laboratory \\
\hline
\end{tabular}


generated using a highly sensitive CCD camera (Princeton Instruments, Evry, France) and the Metaview 4.1.7 image-analysing system (Universal Imaging Corporation).

\section{Results}

The melanogaster subgroup

Mitotic chromosomes hybridized with the Py12 probe and counterstained with DAPI are shown in Figure 1. The heterochromatic structure of the sex chromosomes is presented in Figure 2(a).

The melanogaster complex: The FISH results confirm the paracentromeric position of the ribosomal RNA genes on the $X$ chromosome of $D$. sechellia and $D$. simulans (Figure 1a and $\mathrm{b}$ ) that had previously been described using a unique $28 \mathrm{~S}$ gene probe (Lohe and Roberts, 2000). The Y chromosomes of these two species have different heterochromatic banding patterns, with two large heavily positive DAPI blocks in D. simulans versus four blocks in $D$. sechellia, a finding that is consistent with the quinacrine banding (Lemeunier and Ashburner, 1984) (Figure 2a). The pattern of hybridization on the $\mathrm{Y}$ chromosome is similar in the two species. Repeated observations of highly stretched prometaphase chromosomes show that there are two hybridization sites on the long arm with much lower intensity than the $X$ signal. The location of the signals differs between species: one is close to the tip and the other in a less distal position in D. simulans, one is in the middle of the long arm and the other near the centromere in D. sechellia. The results obtained with the 240-bp repeat probe differ between the species with regard to the signal intensity. On the $\mathrm{X}$ chromosome, the signal is
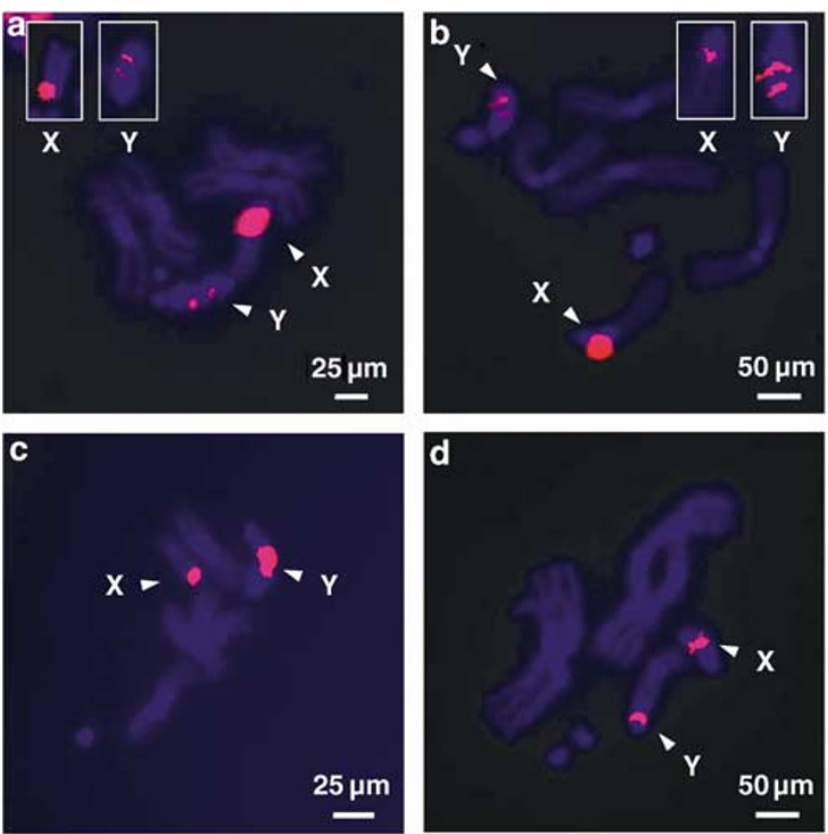

Figure 1 melanogaster subgroup: mitotic chromosomes are counterstained with DAPI and hybridized with the Py12 probe and the 240bp probe (in boxes). (a) D. sechellia, (b) D. simulans, (c) D. santomea and (d) D. yakuba. strong in D. sechellia and weak in D. simulans, whereas the reverse is observed for the $\mathrm{Y}$ hybridization sites (boxes in Figure 1a and b).

In D. mauritiana, the heterochromatic structure of the $\mathrm{Y}$ chromosome differs greatly from that observed in $D$. sechellia and D. simulans (Figure 2a). The Py12 probe produces a strong signal, close to the centromere, on both sex chromosomes (data not shown), which is consistent with the known location of NORs in this species (Lohe and Roberts, 2000).

The $D$. santomea, $D$. yakuba and $D$. teissieri triad: The heterochromatic structure of the $\mathrm{X}$ chromosome, determined after DAPI staining, is very similar in D. santomea and D. yakuba (Figure 2a). A fluorescent heterochromatic block on the long arm of the $X$ chromosome previously described by Lemeunier et al (1978) in D. yakuba is also present in D. santomea, but appears to be fainter, and it does not exist at all in $D$. teissieri. In all three species, the NOR on the $X$ chromosome, determined using the Py12 probe, is in a similar position: on the long arm, and close to the centromere (Figure 1c and d).

The heterochromatic structure of the $\mathrm{Y}$ chromosome is different in $D$. santomea and $D$. yakuba. In the latter species, the short arm of the Y chromosome shows two bright blocks, one close to the centromere, the other at the tip of the short arm, and the long arm presents a short bright block close to the centromere. In D. santomea, the short arm of the Y chromosome shows two bright blocks, which are identical in size and not adjacent to the centromere, and the long arm also has two bright blocks,

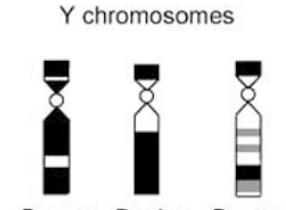

$\begin{array}{lll}\text { D. } \sec & \text { D. } \operatorname{sim} & \text { D. mau }\end{array}$

b ananassae complex

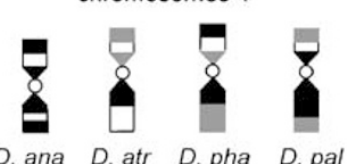

D. ana D. atr D.pha D.pal

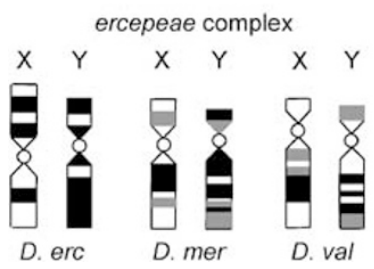

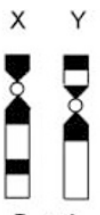

D. yak

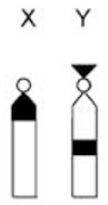

D. $t e i$

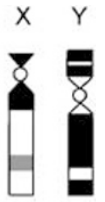

D. san

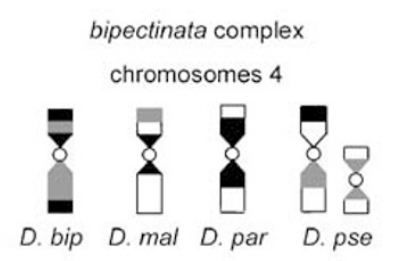

chromosome 4

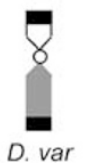

Figure 2 Schematic representation of the heterochromatic structure of the chromosomes bearing an NOR within the melanogaster (a) and the ananassae (b) subgroups. DAPI staining: faint (white box), medium (grey box), intense (black box). (D. sec: D. sechellia, D. sim: D. simulans, D. mau: D. mauritiana, D. yak: D. yakuba, D. tei: D. teissieri, D. san: D. santomea, D. ana: D. ananassae, D. atr: D. atripex, D. pha: D. phaeopleura, D. pal: D. pallidosa, D. bip: D. bipectinata, D. mal: D. malerkotliana, D. par: D. parabipectinata, D. pse: D. pseudoananassae, D. erc: D. ercepeae, D. mer: D. merima, D. val: D. vallismaia, D. var: D. varians.) 
one of them is very large and not adjacent to the centromere, and the other is smaller and terminal. In $D$. teissieri, the observation of stretched chromosomes revealed the existence of a short arm on the $Y$ chromosome, which had never been described before. The long arm carries a bright block in the middle. Hybridization with the Py12 probe showed that the Ylinked NOR lies on the long arm in the three species, close to the centromere in D. santomea and D. yakuba (Figure 1c and d), and lies within the bright block in D. teissieri (data not shown).

\section{The ananassae subgroup}

Mitotic chromosomes hybridized with the Py12 probe and counterstained with DAPI are presented in Figures 3 and 4 . The heterochromatic structure of the sex chromosomes is shown in Figure $2 b$.

The ananassae complex: The four species studied are devoid of NORs on the $X$ chromosome, and $D$. ananassae is the only one to display a NOR on the $\mathrm{Y}$ chromosome (Figure $3 a-c)$. All have an NOR on the metacentric (or submetacentric) chromosome 4 . In $D$. ananassae and $D$. phaeopleura, the hybridization site is terminal, whereas in $D$. atripex and D. pallidosa the site appears to be closer to the centromere. The centromere is always between two heterochromatic blocks, and the NOR is located in a DAPI bright-terminal block. In D. ananassae, there is an additional block at the tip of one arm of chromosome 4, opposite to the arm bearing the NOR (Figure $2 b$ ).

In $D$. atripex and $D$. phaeopleura, we observed differences in the size of the rhodamine signals between the homologues of chromosome 4 (Figure $3 b$ and $c$ ). The differences are strongly marked in D. phaeopleura, for which the largest rhodamine signal is associated with
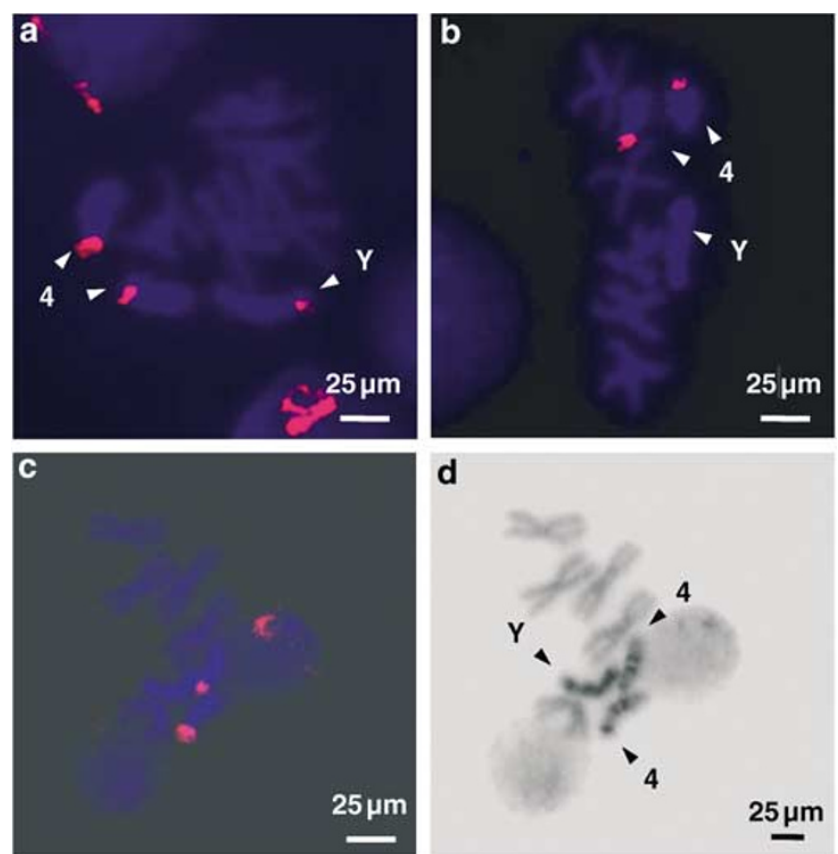

Figure 3 ananassae subgroup (ananassae complex): mitotic chromosomes are counterstained with DAPI and hybridized with the Py12 probe. (a) D. ananassae, (b) D. atripex, (c) D. phaeopleura, (d) D. phaeopleura, DAPI staining with inverted contrast. additional heterochromatic material, as can be observed after DAPI staining (Figure 3c).

The bipectinata complex: The heterochromatic structure of the chromosome 4 and the sex chromosomes is highly variable within the complex (Figure 2b). $D$. pseudoananassae carries an additional and smaller pair of chromosomes 4 . There is only one NOR, located on chromosome 4 (the larger one in D. pseudoananassae), in a telomeric or subtelomeric position (Figure 4c). Differences in the size of the hybridization signals between the homologues are observed in some individuals of $D$. malerkotliana and $D$. parabipectinata (not shown)

The ercepeae complex: In the ercepeae complex, the NORs are located on the sex chromosomes (Figure 4a and $b$ ). In each species, the $X$ chromosome always displays three heterochromatic blocks, the position and intensity of which differ between species (Figure $2 b$ ). The $\mathrm{X}$ chromosome of $\mathrm{D}$. ercepeae is almost metacentric and shows three blocks with the same intensity of DAPI staining. The NOR is located in the middle of the arm of the $X$ chromosome, which has a single DAPI-positive block. In D. vallismaia and D. merina, the NOR is situated on a weaker DAPI band, close to the centromere, but on different arms (the long arm of the $X$ chromosome in D. vallismaia, and the short arm in D. merina).

The $\mathrm{Y}$ chromosomes of $D$. vallismaia and $D$. merina are both submetacentric and their banding patterns display some similarities. They show two hybridization signals of the Py12 probe, differing in size, one at each tip of the chromosome (Figure 4a). The $\mathrm{Y}$ chromosome of $D$. ercepeae appears slightly different in its heterochromatic banding pattern. It is characterized by a unique
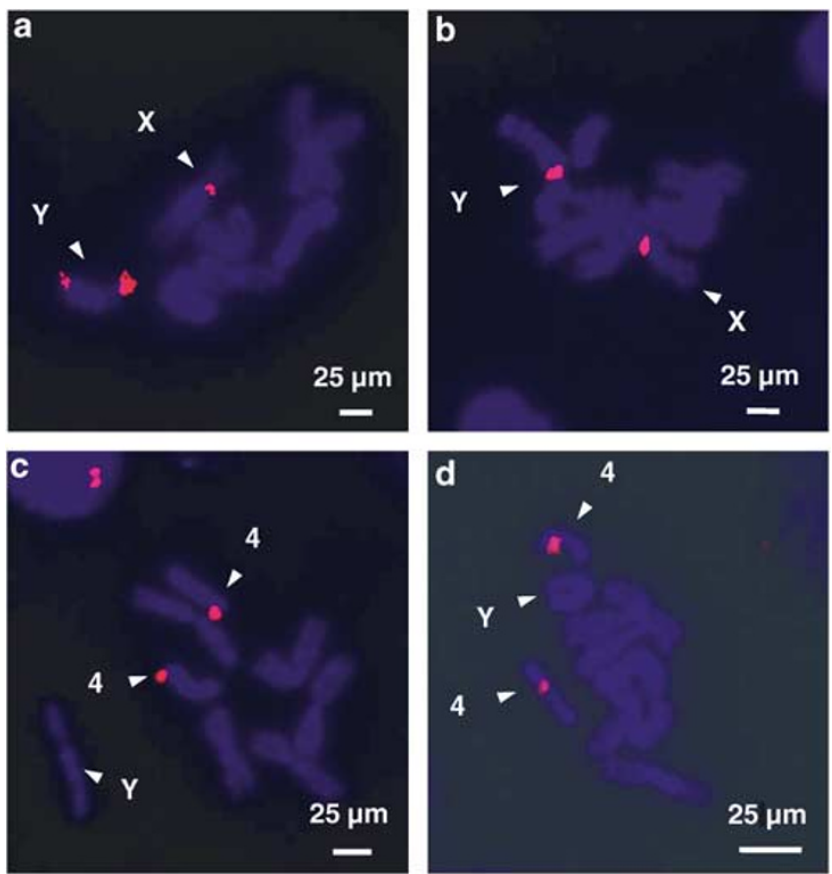

Figure 4 ananassae subgroup: mitotic chromosomes are counter stained with DAPI and hybridized with the Py12 probe. (a) D. vallismaia, (b) D. ercepeae (ercepeae complex), (c) D. malerkotliana (bipectinata complex), (d) D. varians. 
hybridization site, in a distal position on the long arm (Figure $4 b$ ).

$D$. varians: D. varians possesses sex chromosomes and a chromosome 4, which have heterochromatic banding patterns very different from those of the species belonging to the three complexes (Figure $2 b$ ). D. varians has a unique NOR, situated on the shorter arm of the submetacentric chromosome 4, close to the centromere (Figure 4d). In some individuals, we observed differences in the sizes of the signals between the homologues.

\section{Discussion}

rDNA evolution and Drosophila phylogeny

In the light of the phylogenetic relationships previously established in the melanogaster group, we can see how the data reported here help to clarify the placement of the species studied. This leads us to propose the diagram presented in Figure 5, which shows the most parsimonious scenario for NOR evolution, as detailed below.

In the melanogaster subgroup, the relationships among the sibling species $D$. simulans, $D$. sechellia and D. mauritiana are still obscure. The allozymic data (Cariou, 1987) and introgression experiments (Palopoli et al, 1996) tend to group D. simulans and D. sechellia together. The assigning of D. sechellia and D. mauritiana to the same group is supported by DNA-DNA hybridization and gene sequence data (Caccone et al, 1996). Finally, phylogenic reconstruction using microsatellites provides evidence that $D$. sechellia arose first, followed by a split between $D$. simulans and D. mauritiana (Harr et al, 1998). The data on NORs (Lohe and Roberts, 2000) and the present findings strengthen the hypothesis that $D$. sechellia is the closest relative of $D$. simulans. Not only do both these species have no NOR on the Y chromosome, they also reveal two weak hybridization sites on this chromosome with the Py12 probe. The same pattern was observed in two other stocks of $D$. simulans from different geographic locations (unpublished data). The hybridization with the 240 -bp repeat probe in $D$. simulans

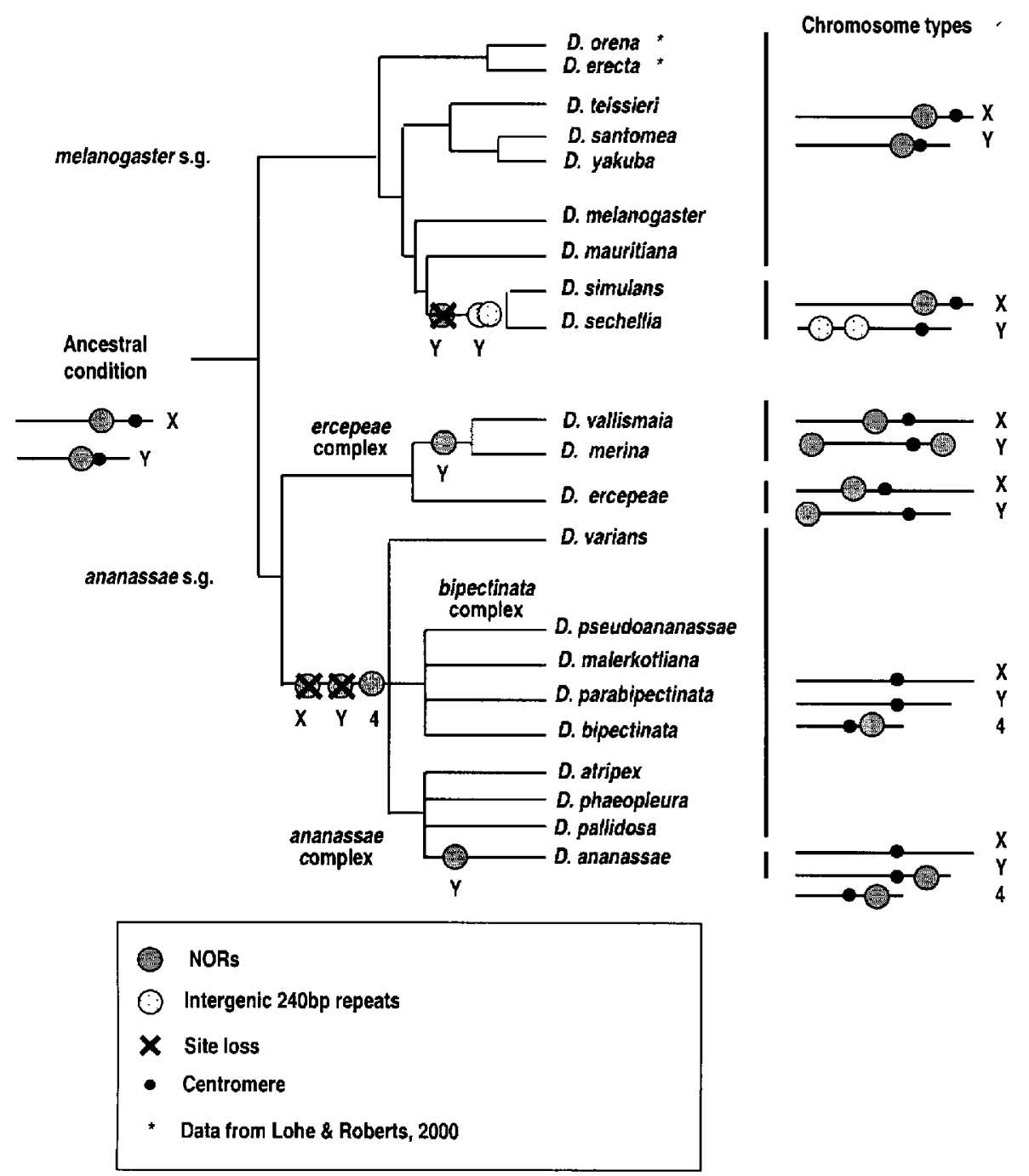

Figure 5 Parsimonious scenario for NORs' evolution (see text). When the relationships between species are not well supported by data of the literature, these are represented as unresolved branching. Chromosome types represent the different NOR configurations observed across species. 
shows that both sites result from an amplification of this sequence (shown by the very strong signal). In $D$. sechellia, the signal on the $Y$ chromosome with this probe remains very weak, whereas the signal on the $X$ chromosome is still strong. This suggests that there has been an amplification of intergenic sequences on the $X$ chromosome, but not on the $\mathrm{Y}$ chromosome. Lohe and Roberts (2000), using a $28 \mathrm{~S}$ probe, failed to detect any hybridization signal on the $Y$ chromosome of $D$. sechellia, which suggested a complete or nearly complete loss of the coding sequences. Whether sequences other than the 240 -bp repeats are present on this chromosome remains an open question. The most parsimonious scenario is that the events involving the loss of the Y-linked NOR and the acquisition of a second site possessing rDNArelated sequences on the $\mathrm{Y}$ chromosome occurred only once. This would mean that D. sechellia and D. simulans originated from a common ancestor after the split that led to D. mauritiana. The difference in the hybridization signal size between the two species would therefore result from heterochromatin rearrangements after the speciation event.

In the ananassae subgroup, only the species belonging to the ercepeae complex were found to carry NORs on both sex chromosomes, which is the general pattern in the melanogaster subgroup. We propose that this pattern is ancestral in the melanogaster group, and that the ercepeae complex was the first to evolve within the subgroup. Although the evolutionary scheme we propose needs to be confirmed by further studies, we can already see that it is consistent with recent data on gene sequence divergence (Schawaroch, 2002; Da Lage, personal communication). The loss of the Y- and Xlinked NORs, and the acquisition of an NOR on the fourth chromosome, would seem to have occurred in the lineage leading to the ananassae and bipectinata complexes, before their split. Owing to the major heterochromatic rearrangements that have occurred, we cannot, however, exclude the possibility that an NOR may have been acquired by chromosome 4 several times independently. According to our scenario, the presence of a Y-linked NOR in D. ananassae results from a secondary acquisition. The absence of a Y-linked NOR in $D$. pallidosa, which is thought to be its closest relative (Singh, 2000), supports this hypothesis. This would imply that the NOR on the fourth chromosome did not necessarily arise as a result of transposition from the $X$ chromosome, as proposed by Hinton and Downs (1975), but may have originated from the $\mathrm{Y}$ chromosome.

Within the ercepeae complex, the $Y$ chromosomes of $D$. vallismaia and $D$. merina appear to be structurally very similar to each other and also both bear two rDNA hybridization sites, whereas the $\mathrm{Y}$ chromosome of $D$. ercepeae showed a single site. $D$. vallismaia and D. merina appear to be the closest relatives in this triad, as judged from their ability to produce fertile hybrids, from the structure of the autosomes and X chromosome, and from morphological and molecular data (Lemeunier et al, 1997; Da Lage, personal communication). If they are, then the shared pattern observed in D. vallismaia and D. merina would require at least two events: either two independent acquisitions of a Y-linked NOR or the acquisition of a Y-linked NOR in the lineage leading to the three species, followed by the secondary loss of a Y-linked NOR in the lineage leading to $D$. ercepeae. This latter hypothesis, which requires $D$. vallismaia and $D$. merina to be the closest relatives, is more parsimonious because it requires only one acquisition of NOR.

The phylogenetic position of $D$. varians is still unclear. Its inclusion in the ananassae subgroup was initially challenged, on the basis of some characteristics of its periphallic organs (Bock and Wheeler, 1972). Recent molecular data support this assignation, but do not enlighten us about the relationships of this species with the three complexes (Schawaroch, 2002). The structure of its mitotic chromosomes, which have been examined here, does not provide any further information about its possible position within the subgroup. However, the presence of an NOR on its fourth chromosome tends to bring $D$. varians closer to the bipectinata and ananassae complexes.

\section{Functional constraints on the chromosomal location of NORs?}

Although sequences unrelated to rDNA might also be involved (Park and Yamamoto, 1995), cytological and molecular studies in D. melanogaster have produced evidence that the NORs, and more precisely the 240-bp repeats of the intergenic spacer, function as $\mathrm{X}-\mathrm{Y}$ pairing sites during meiosis (McKee and Karpen, 1990; McKee et al, 1992). In this context, Lohe and Roberts (2000) argued that the persistence of NORs on both sex chromosomes within the melanogaster subgroup results from this functional constraint. The cytological observations of Ault and Rieder (1994) strongly suggest that the 240-bp repeats on the $Y$ chromosome of $D$. simulans do play this role, thus compensating for the lack of a Y-linked NOR in this species. Our study shows that the situation on the $\mathrm{Y}$ chromosome of $D$. sechellia may be similar to that in $D$. simulans. The situation in the melanogaster subgroup is consistent with a role for NORs in pairing, whereas their apparent absence from the sex chromosomes in species of the bipectinata and ananassae complexes (except D. ananassae, which carries a Y-linked NOR) conflicts with this hypothesis. The sequences of the rDNA genes have been proven to be highly conserved during evolution and, moreover, we obtained strong hybridization signals on the fourth chromosome of these species, with the Py12 plasmid cloned in D. melanogaster. Thus, the lack of signal on the sex chromosomes can be definitely ascribed to a lack of rDNA genes. We also tested one species of each complex with the 240-bp repeat probe from $D$. simulans (data not shown). No signal was detected, even at the NOR location on the fourth chromosome, which strongly suggests a marked divergence of this sequence at the species group level. This means that we cannot exclude the possibility that intergenic spacer repeats are present on the sex chromosomes of the ananassae subgroup species, and function as pairing sites. Observations of multivalents composed of the $\mathrm{X}, \mathrm{Y}$ and fourth chromosomes during male meiosis in $D$. ananassae (Hinton and Downs, 1975; Matsuda et al, 1983) are consistent with this hypothesis. Although the $\mathrm{X}$ chromosome is devoid of NORs in this species, it has been seen to pair with other chromosomes at sites that may correspond to their NORs (figures in Tobari et al, 1993). Further studies will require the use of spacer probes cloned from the ananassae subgroup. 
Heterochromatin and rDNA evolution

The changes in the number and location of NORs among Drosophila species studied at the group scale have proved to be quite revealing about the rearrangements affecting heterochromatin. This is particularly clear for the $\mathrm{X}$ linked NORs. These have a similar paracentromeric position in all but one of the nine species of the melanogaster subgroup, a situation that can be linked to the limited degree of heterochromatic changes on this chromosome. D. orena is an exception, in that it has acquired a considerable amount of heterochromatic material; a change that was accompanied by a change in NOR location (Lemeunier et al, 1978). In contrast, within the ananassae subgroup, the $X$ chromosome is characterized by appreciable heterochromatic rearrangements. Only the ercepeae complex has retained an Xlinked NOR, the position of which differs considerably between species. The positions of the Y-linked NORs, which have sometimes been lost and sometimes duplicated, are highly variable in both subgroups. This is consistent with the major organizational changes undergone by the $\mathrm{Y}$ chromosome, which is entirely heterochromatic. Even at the species-complex scale, the banding pattern of this chromosome is never maintained.

In D. melanogaster, as in many other species, the fourth chromosome appears as a small dot chromosome that is considered atypical compared to the other autosomes, mainly because of its heterochromatic structure. In the ananassae subgroup, as shown here, and in some other species, such as D. kikkawai (Baimai and Chumchong, 1980) and D. birchii (Baimai, 1969), the fourth chromosome is large and metacentric, and an additional pair of heterochromatic chromosomes 4 is sometimes observed. In several species of the ananassae subgroup, we observed size differences in NOR signals between the homologous chromosomes 4 . The case of $D$. phaeopleura is particularly remarkable, with a translocation of a part of the tip, bearing an NOR, from one chromosome to its homologue, and the presence in some individuals of a supernumerary chromosome 4 . The heteromorphism of homologous NORs has been well documented both in animals (Delany et al, 1991; Abuin et al, 1996; Hirai et al, 1998; Mandrioli et al, 1999) and plants (Miller et al, 1980; Moscone et al, 1995; Zoldos et al, 1999). This may be related to differences in the ribosomal gene copy number at homologous sites, which has been attributed to unequal crossing over (Fitch et al, 1990; Dover et al, 1993; Delany and Krupkin, 1999; Mandrioli et al, 1999). In $D$. melanogaster, several lines of evidence suggest that the chromosome 4 may have derived from an ancestral $X$ chromosome (Hochman, 1976; Larsson et al, 2001). So far, few studies have investigated the nature of the rearrangements that have affected the fourth chromosome. Further investigation of this issue should help to elucidate the role of heterochromatin in the process of chromosome evolution.

\section{Acknowledgements}

We would like to thank Dr Lohe for kindly providing a 240-bp probe, and D Ogereau for the preparation of the probes.

\section{References}

Abuin M, Clabby C, Martinez P, Goswami U, Flavin F, Wilkins NP et al (1996). A NOR-associated repetitive element present in the genome of two Salmo species (Salmo salar and Salmo trutta). Genome 39: 671-679.

Andronico F, De Lucchini S, Graziani F, Nardi I, Batistoni R, Barsacchi-Pilone G (1985). Molecular organization of ribosomal RNA genes clustered at variable chromosomal sites in Triturus vulgaris meridionalis (Amphibia, Urodela). J Mol Biol 186: 219-229.

Ault JG, Rieder CL (1994). Meiosis in Drosophila males. I. The question of separate conjunctive mechanisms for the $\mathrm{XY}$ and autosomal bivalents. Chromosoma 103: 352-356.

Baimai V (1969). Karyotype variation in Drosophila birchii. Chromosoma 27: 381-394.

Baimai V, Chumchong C (1980). Karyotype variation and geographic distribution of the three sibling species of the Drosophila kikkawai complex. Genetica 54: 113-120.

Bicudo H (1981). Further observation on the nucleolar organizing activity in salivary gland cells of Drosophila mulleri, Drosophila arizonensis and their hybrids. Biol Zentralbl 100: 597-612.

Bock IR, Wheeler MR (1972). The Drosophila melanogaster species group. Univ Tex Publ 7213: 1-102.

Caccone A, Moriyama EN, Gleason JM, Nigro L, Powell JR (1996). A molecular phylogeny for the Drosophila melanogaster subgroup and the problem of polymorphism data. Mol Biol Evol 13: 1224-1232.

Cariou ML (1987). Biochemical phylogeny of the eight species in the Drosophila melanogaster subgroup, including D. sechellia and D. orena. Genet Res Camb 50: 181-185.

Delany ME, Krupkin AB (1999). Molecular characterization of ribosomal gene variation within and among NORs segregating in specialized populations of chicken. Genome 42: 60-71.

Delany ME, Muscarella DE, Bloom SE (1991). Formation of nucleolar polymorphisms in trisomic chickens and subsequent microevolution of rRNA gene clusters in diploids. J Hered 82: 213-220.

Dover GA, Linares AR, Bowen T, Hancock JM (1993). The detection and quantification of concerted evolution and molecular drive. Methods Enzymol 224: 525-541.

Dubcovsky J, Dvorak J (1995). Ribosomal RNA multigene loci: nomads of the Triticeae genomes. Genetics 140: 1367-1377.

Fitch DH, Strausbaugh LD, Barrett V (1990). On the origins of tandemly repeated genes: does histone gene copy number in Drosophila reflect chromosomal location? Chromosoma 99: 118-124.

Galian J, Serrano J, De la Rua P, Petitpierre E, Juan C (1995). Localization and activity of rDNA genes in tiger beetle (Coleoptera: Cicindelinae). Heredity 74: 524-530.

Harr B, Weiss S, David JR, Brem G, Schlotterer C (1998). A microsatellite-based multilocus phylogeny of the Drosophila melanogaster species complex. Curr Biol 8: 1183-1186.

Hennig W, Link B, Leoncini O (1975). The location of the nucleolus organizer regions in Drosophila hydei. Chromosoma 51: 57-63.

Hennig W, Vogt P, Jacob G, Siegmund I (1982). Nucleolus organizer regions in Drosophila species of the repleta group. Chromosoma 87: 279-292.

Hinton CW, Downs JE (1975). The mitotic, polytene, and meiotic chromosomes of Drosophila ananassae. J Hered 66: 353-361.

Hirai H, Hasegawa Y, Kawamoto Y, Tokita E (1998). Tandem duplication of nucleolus organizer region (NOR) in the Japanese macaque, Macaca fuscata fuscata. Chromosome Res 6: 191-197.

Hirai H, Yamamoto MT, Taylor RW, Imai HT (1996). Genomic dispersion of $28 \mathrm{~S}$ rDNA during karyotypic evolution in the ant genus Myrmecia (Formicidae). Chromosoma 105: 190-196. 
Hochman B (1976). The fourth chromosome of Drosophila melanogaster. In: Ashburner M, Novitski E (eds) The Genetics and Biology of Drosophila. Academic Press: London. Vol 1b, pp 903-928.

John B, Miklos G (1988). The Eukaryote Genome in Development and Evolution. Allen and Unwin: Boston, Sydney, Wellington.

Kaufmann BP (1937). Morphology of the chromosomes of Drosophila ananassae. Cytologia Fujii Jubilee Volume: 1043-1055.

Kikkawa H (1938). Studies on the genetics and cytology of Drosphila ananassae. Genetica 20: 458-516.

Knibb WR, Barker JS, Oakeshott JG (1989). The genetics of abnormal abdomen, incomplete abdomen, and bobbed in Drosophila buzzatii. Genome 32: 754-761.

Larsson J, Chen JD, Rasheva V, Rasmuson-Lestander A, Pirrotta V (2001). Painting of fourth, a chromosome-specific protein in Drosophila. Proc Natl Acad Sci USA 98: 6273-6278.

Lemeunier F, Ashburner M (1984). Relationships within the melanogaster species subgroup of the genus Drosophila (Sophophora). IV. The chromosomes of two new species. Chromosoma 89: 343-351.

Lemeunier F, Aulard S, Arienti M, Jallon JM, Cariou ML, Tsacas L (1997). The ercepeae complex: new cases of insular speciation within the Drosophila ananassae species subgroup (melanogaster group) and descriptions of two new species (Diptera: Drosophilidae). Ann Ent Soc Am 90: 28-42.

Lemeunier F, Dutrillaux B, Ashburner M (1978). Relationship within the melanogaster subgroup species of the genus Drosophila (Sophophora). III The mitotic chromosomes and quinacrine fluorescent patterns of polytenes chromosomes. Chromosoma 69: 349-361.

Lemeunier F, Tsacas L, David J, Ashburner M (1986). The melanogaster species group. In: Ashburner M, Carson HL, Thompson Jr JN (eds) The Genetics and Biology of Drosophila. Academic Press: London. Vol 3e, pp 147-256.

Lohe AR, Roberts PA (1990). An unusual Y chromosome of Drosophila simulans carrying amplified rDNA spacer without rRNA genes. Genetics 125: 399-406.

Lohe AR, Roberts PA (2000). Evolution of DNA in heterochromatin: the Drosophila melanogaster sibling species subgroup as a resource. Genetica 109: 125-130.

Mandrioli M, Manicardi GC, Bizzaro D, Bianchi U (1999). NOR heteromorphism within a parthenogenetic lineage of the aphid Megoura viciae. Chromosome Res 7: 157-162.

Matsuda M, Imai H, Tobari YN (1983). Cytogenetic analysis of recombination in males of Drosophila ananassae. Chromosoma 88: 286-292.

McKee BD, Habera L, Vrana JA (1992). Evidence that intergenic spacer repeats of Drosophila melanogaster rRNA genes func- tion as $\mathrm{X}-\mathrm{Y}$ pairing sites in male meiosis, and a general model for achiasmatic pairing. Genetics 132: 529-544.

McKee BD, Karpen GH (1990). Drosophila ribosomal RNA genes function as an $\mathrm{X}-\mathrm{Y}$ pairing site during male meiosis. Cell 61: 61-72.

Miller TE, Gerlach WL, Flavell RB (1980). Nucleolus organiser variation in wheat and rye revealed by in situ hybridization. Heredity 45: 377-382.

Moscone EA, Loidl J, Ehrendorfer F, Hunziker AT (1995). Analysis of active nucleolar organizing regions in Capsicum (Solanaceaea) by silver staining. Am J Bot 82: 276-287.

Palopoli MF, Davis AW, Wu CI (1996). Discord between the phylogenies inferred from molecular versus functional data: uneven rates of functional evolution or low levels of gene flow? Genetics 144: 1321-1328.

Park HS, Yamamoto MT (1995). The centric region of the $\mathrm{X}$ chromosome rDNA functions in male meiotic pairing in Drosophila melanogaster. Chromosoma 103: 700-707.

Powell JR (1997). Progress and Prospects in Evolutionary Biology: The Drosophila Model. Oxford University Press, Inc.: New York.

Schawaroch V (2002). Phylogeny of a paradigm lineage: the Drosophila melanogaster species group (Diptera: drosophilidae). Biol J Linn Soc 76: 21-37.

Shishido R, Sano Y, Fukui K (2000). Ribosomal DNAs: an exception to the conservation of gene order in rice genomes. Mol Gen Genet 263: 586-591.

Singh BN (2000). Drosophila ananassae: a species characterized by several unusual genetics features. Curr Sci 78: 391-398.

Sinibaldi RM, Cummings MR (1981). Localization and characterization of rDNA in Drosophila tumiditarsus. Chromosoma 81: 655-671.

Stuart WD, Bishop JG, Carson HL, Frank MB (1981). Location of the 18/28S ribosomal RNA genes in two Hawaiian Drosophila species by monoclonal immunological identification of RNA. DNA hybrids in situ. Proc Natl Acad Sci USA 78 3751-3754.

Templeton AR, Crease TJ, Shah F (1985). The molecular through ecological genetics of abnormal abdomen in Drosophila mercatorum. I. Basic genetics. Genetics 111: 805-818.

Tobari YN, Goni B, Tomimura Y, Matsuda M (1993). Chromosomes. In: Tobari YN (ed) Drosophila ananassae - Genetical and Biological Aspects. Japan Scientific Societies Press: Tokyo and Karger, Basel. pp 23-48.

Zoldos V, Papes D, Cerbah M, Panaud O, Besendorfer V, SiljakYakovlev S (1999). Molecular-cytogenetic studies of ribosomal genes and heterochromatin reveal conserved genome organization among 11 Quercus species. Theor Appl Genet 806: 1-9. 